\title{
Folate-related polymorphisms in gastrointestinal stromal tumours: susceptibility and correlation with tumour characteristics and clinical outcome
}

\author{
Sabrina Angelinii, ${ }^{\star}$, Gloria Ravegnini ${ }^{1,7}$, Margherita Nannini ${ }^{2,7}$, Justo Lorenzo Bermejo ${ }^{3}$, Muriel Musti ${ }^{4}$, \\ Maria A Pantaleo ${ }^{2}$, Elena Fumagalli ${ }^{5}$, Nicola Venturoli ${ }^{6}$, Elena Palassini ${ }^{5}$, Nicola Consolini ${ }^{1}$, Paolo G Casali ${ }^{5}$, \\ Guido Biasco $^{2,8}$ and Patrizia Hrelia ${ }^{1,8}$
}

The folate metabolism pathway has a crucial role in tumorigenesis as it supports numerous critical intracellular reactions, including DNA synthesis, repair, and methylation. Despite its importance, little is known about the influence of the folate pathway on gastrointestinal stromal tumour (GIST), a rare tumour with an incidence ranging between 6 and 19.6 cases per million worldwide. The importance of folate metabolism led us to investigate the influence of polymorphisms in the genes coding folate-metabolising enzymes on GIST susceptibility, tumour characteristics and clinical outcome. We investigated a panel of 13 polymorphisms in 8 genes in 60 cases and 153 controls. The TS 6-bp deletion allele (formerly rs34489327, delTInsTTAAAG) was associated with reduced risk of GIST $(O R=0.20,95 \% \mathrm{Cl} 0.05-0.67, P=0.0032)$. Selected polymorphisms in patients stratified by age, gender, and other main molecular and clinical characteristics showed that few genotypes may show a likely correlation. We also observed a significant association between the RFC AA/AG genotype and time to progression $(H R=0.107$, $95 \% \mathrm{Cl} 0.014-0.82 ; P=0.032$ ). Furthermore, we observed a tendency towards an association between the SHMT1 variant allele (TT, rs 1979277) and early death ( $\mathrm{HR}=4.53,95 \% \mathrm{Cl} 0.77-26.58, P=0.087)$. Aware of the strengths and limitations of the study, these results suggest that polymorphisms may modify the risk of GIST and clinical outcome, pointing to the necessity for further investigations with information on folate plasma levels and a larger study population.

European Journal of Human Genetics (2015) 23, 817-823; doi:10.1038/ejhg.2014.198; published online 17 September 2014

\section{INTRODUCTION}

Folate metabolism supports numerous critical intracellular reactions, including DNA synthesis, repair, and methylation. DNA methylation status is essential for normal development and maintenance of cellular homeostasis and functions in adult organisms, including silencing of repetitive DNA elements, proper expression of genetic information and maintenance of genomic structural integrity. ${ }^{1}$ The accurate maintenance of DNA synthesis, repair, and methylation patterns is fundamental, and its balance in mature cells is maintained by the concerted action of more than 30 enzymes. ${ }^{2}$ These enzymes are highly polymorphic, and several functional genetic polymorphisms have been attracting research interest, as they may be responsible for altered DNA synthesis, repair and methylation processes, all of which are crucial in relation to carcinogenesis. Aberrant methylation patterns have been associated with various diseases including cancer and neurodegenerative diseases. ${ }^{3,4}$ In addition, polymorphisms in genes involved in the folate metabolisms have been associated with cancer risk, including colorectal and gastric cancer, vascular disease, depression, and Down's syndrome. ${ }^{5}$ Currently, there are evidences of an association between DNA methylation level and polymorphisms in folate metabolism genes. ${ }^{6,7}$
A recent finding suggests that the DNA methylation profile may be associated with aggressive clinical behaviour and unfavourable prognosis in gastrointestinal stromal tumours (GISTs). ${ }^{8}$ However, as far as we are aware, there have been no investigations exploring the influence of genetic polymorphisms in enzymes that take part in the folate metabolic pathways in GIST. GIST represents the most common mesenchymal tumour of the gastrointestinal tract, characterised by KIT or platelet-derived growth factor receptor $\alpha$ (PDGFRA) activating mutations. ${ }^{9,10}$ In particular, herein, we analysed the frequencies of genetic polymorphisms - two insertion/deletion, one tandem repeat, and ten single-nucleotide polymorphisms - in eight genes belonging to the folate pathway, in GIST patients and controls. Specific aims of this study are to test if these polymorphisms: (i) influence the risk of developing GIST, or (ii) are associated with specific patients' characteristics and clinical features of their tumours. Furthermore, the importance of the folate metabolic pathway in genomic stability maintenance, along with the fact that secondary point mutations in KIT or PDGFRA genes are one of the mechanisms of acquired resistance in GIST patients undergoing imatinib therapy, has prompted us to evaluate the influence of the selected polymorphisms on imatinib response. Finally, alterations in the folate metabolic

${ }^{1}$ Department of Pharmacy and Biotechnology, University of Bologna, Bologna, Italy; ${ }^{2}$ Department of Specialized, Experimental and Diagnostic Medicine, Sant'Orsola-Malpighi Hospital, University of Bologna, Bologna, Italy; ${ }^{3}$ Institute of Medical Biometry and Informatics, University of Heidelberg, Heidelberg, Germany; ${ }^{4}$ Department of Public Health, Epidemiological Service, Local Health Authority of Bologna, Bologna, Italy; ${ }^{5}$ Adult Mesenchymal Tumour Medical Oncology Unit, Istituto Nazionale Tumori, Milano, Italy; ${ }^{6}$ Immunohematology and Blood Transfusion, Sant'Orsola-Malpighi Hospital, University of Bologna, Bologna, Italy

*Correspondence: Professor S Angelini, Department of Pharmacy and Biotechnology, Via Irnerio 48, 40126 Bologna, Italy. Tel:+39 051 2091787 ; Fax:+39 051 2091780;

E-mail: s.angelini@unibo.it

${ }^{7}$ These authors contributed equally to this work

8These two authors jointly directed this work.

Received 19 November 2013; revised 7 August 2014; accepted 15 August 2014; published online 17 September 2014 
pathway may induce aberrant methylation patterns that might be associated with disease progression. In light of this a further aim of the study will be to correlate the presence of polymorphisms with the patients' overall survival (OS).

\section{MATERIALS AND METHODS}

\section{Study population}

A total of 60 unresectable/metastatic GIST patients were retrospectively enroled in this study from 2004 to 2008. Thirty-two patients were enroled at the Sant'Orsola-Malpighi Hospital, Bologna, and 28 at the Istituto Nazionale dei Tumori, Milan, Italy. Clinical information were collected retrospectively from the patients' medical records. OS was defined as the time from the first day of treatment to death from disease. Dates of death were obtained and cross-checked using the inpatient medical records. If a patient was alive, OS was censored at the time of the last follow-up. For the 54 patients on standard first-line imatinib therapy time to progression (TTP) was calculated from the start of imatinib therapy to the date of disease progression documented by CT scan performed approximately every 3-4 months. TTP for patients who did not progress was calculated by censoring at the time of last follow-up. In order to exclude disease susceptibility we also genotyped 153 controls, anonymous blood donors from the Centro Trasfusionale, Sant'Orsola-Malpighi Hospital, Bologna, recruited in 2007-2008. The study was approved by the Ethics Committees of the two institutions. The analysis was done after written informed consent for study participation and anonymous data publication in accordance with national legislation. Any subject could cancel participation at any time during the study, according to the Helsinki Declaration and later Amendments.

\section{Genotyping analysis}

We selected 13 common (minor allele frequency (MAF) $>0.05$ in Caucasians), well-studied functional variants - located in the regulatory region, causing nonsynonymous amino-acid changes, and/or having been repeatedly associated with cancer risk, survival or treatment response. Patients with available peripheral blood were eligible for this retrospective study. DNA was extracted from fresh or frozen whole blood using a DNA isolation kit from Qiagen (QIAamp DNA Mini Kit, Qiagen, Hilden, Germany). Characteristics of the studied polymorphisms - two insertion/deletion, one tandem repeat, and ten single-nucleotide polymorphisms - are reported in Supplementary Table S1. Genotypes were determined by polymerase chain reaction (PCR)-based assays (restriction fragment length polymorphism (RFLP) and/or real-time) according to published methods. ${ }^{11-14}$ or as recommended by the manufacturer. Positive and negative controls were included in each reaction as quality control. In addition, for internal quality control (accuracy of genotyping) 90\% of samples were repeated. The concordance between the original and the duplicate samples for all the analysed polymorphisms was $100 \%$.

\section{Statistical analysis}

The distribution of genotypes was tested for departures from Hardy-Weinberg equilibrium using $\chi^{2}$-test. For continuous variables, medians were compared using Wilcoxon rank-sum test; the frequency distributions of categorical variables were compared using Fisher's exact test. Survival analysis methods were used to examine the relationship between genotypes (homozygous wild type, heterozygous and homozygous for the variant allele) and GIST TTP. The survival functions were estimated with Kaplan-Meier estimates and compared using log-rank tests. Univariate and multivariate Cox regression analyses were fitted for the outcomes (adjusting for gender, age, and status (localised/ metastatic) at diagnosis). The proportional hazards assumption was tested $(P>0.05)$ using Schoenfeld residuals. Exact logistic regression (adjusting for gender and age) was used to assess the relation between individual polymorphisms and primary resistance. Follow-up started with patient diagnosis for OS and the TTP. Given the limited sample size of the present study, probability values and additional parameter estimates were not adjusted for multiplicity. Results should be interpreted as exploratory. $P$-values $\leq 0.05$ were considered significant. Genotyping data are available at http://www.ncbi.nlm.nih.gov/ clinvar, with accession number from SCV000187672 to SCV000187683. Statistical analysis was conducted using Stata Intercooled version 12.0. ${ }^{15}$

\section{RESULTS}

\section{Characteristics of the study population}

The cohort of 60 Caucasian GIST patients (Supplementary Table S2) was made up of $65 \%$ men and $35 \%$ women (median age at diagnosis 58.0 years; range $18-83$ years). The most common primary sites were the stomach (55.0\%) and the small intestine $(40.0 \%)$; in the remaining $5 \%$, the sites of onset were oesophagus (1 case) and rectum (2 cases). Twenty-three patients $(38.3 \%)$ had metastatic disease, with the liver and the peritoneum being the most common sites of metastases. Thirty-five patients (58.3\%) harboured KIT exon 11 mutations, whereas only one patient had a KIT exon 9 mutation. Three patients (5.6\%) had a PDGFR $\alpha$ mutation, excluding the c.2525A $>\mathrm{T}$, p.(Asp842Val), and 9 (15.0\%) were KIT/PDGFR $\alpha$ WT GISTs. In 12 GISTs, mutational status was unknown, owing to insufficient or unavailable biological material for the analysis. The control group $(n=153)$ was made up of $60 \%$ men and $40 \%$ women (median age 47.0 years, range $21-79$ years).

\section{Genotype distribution in the two studied populations}

Genotype frequencies of the 13 polymorphisms were found to be in Hardy-Weinberg equilibrium $(P>0.05)$ in both patients and controls, with the exception of rs1801133 (Chr1.hg.11:g.11796321G >A) in the MTHFR genes in controls only $(P=0.016)$. MAF and Hardy-Weinberg equilibrium $P$-value are presented in Supplementary Table S3.

Details of the results of association tests for each genetic polymorphism chosen for analysis in our case-control study population are presented in Table 1. The most significant result was found for the 6-bp ins/del in TS gene (formerly rs34489327, delTInsTTAAAG). This SNP was associated with GIST risk $(P=0.0032)$. In particular the 6- bp del/del genotype was associated with a decreased susceptibility to GIST (OR $=0.20,95 \%$ CI $0.05-0.67$, Table 1$)$. There was no significant difference in the genotype distribution or allele frequencies between cases and controls for any of the other polymorphisms tested across the key enzymes that take part in the folate metabolic pathways.

\section{Association between genetic polymorphisms and clinical features at diagnosis}

With regard to mutational status, we divided the patients into three groups - KIT or PDGFRA mutated and wt GIST. The most relevant results are presented in Table 2. According to this stratification, univariate analysis showed an excess of wild-type RFC genotype in patients with PDGFRA mutations compared with KIT mutated and wild-type GIST (100\% vs $38.9 \%$ and $11.1 \%$, respectively; $P=0.028$ ). We also observed that the TS 2R2R genotype (rs45445694) was more represented in PDGFRA-mutated patients compared with KIT mutated and wild-type GIST ( $66.7 \%$ vs $16.7 \%$ and $0 \%$, respectively; $P=0.034)$. Another finding was an excess of the presence of at least a variant MTRR rs10380 allele in wild-type GIST compared with KIT/PDGFRA-mutated patients $(33.3 \%$ vs $2.8 \%$ and $0 \%$, respectively; $P=0.033)$. The same finding was observed for MTRR rs162036 (44.0\% vs $8.3 \%$ and $0 \%$, respectively; $P=0.032$ ). An association was also seen for MTHFR (rs1801133) genotype. In particular we observed an excess of the wild-type genotype in PDGFRA-mutated and wildtype GIST compared with KIT-mutated patients $(66.7 \%$ both $v s$ $27.8 \% ; P=0.036$ ). None of the other polymorphisms analysed was correlated with tumour mutational status (data not shown).

Exact Logistic regression showed that of the 28 cases with at least one RFC variant allele, $9(32.1 \%)$ had a tumour size larger than $10 \mathrm{~cm}$. In contrast, of the 19 GIST patients with a wt RFC, 13 (68.4\%) had a tumour size larger than $10 \mathrm{~cm}$. The odds ratio for the probability of a tumour size larger than $10 \mathrm{~cm}$ in individuals with at least one variant 
Table 1 Genotype distribution of polymorphisms in genes belonging to the folate pathway in GIST patients and controls

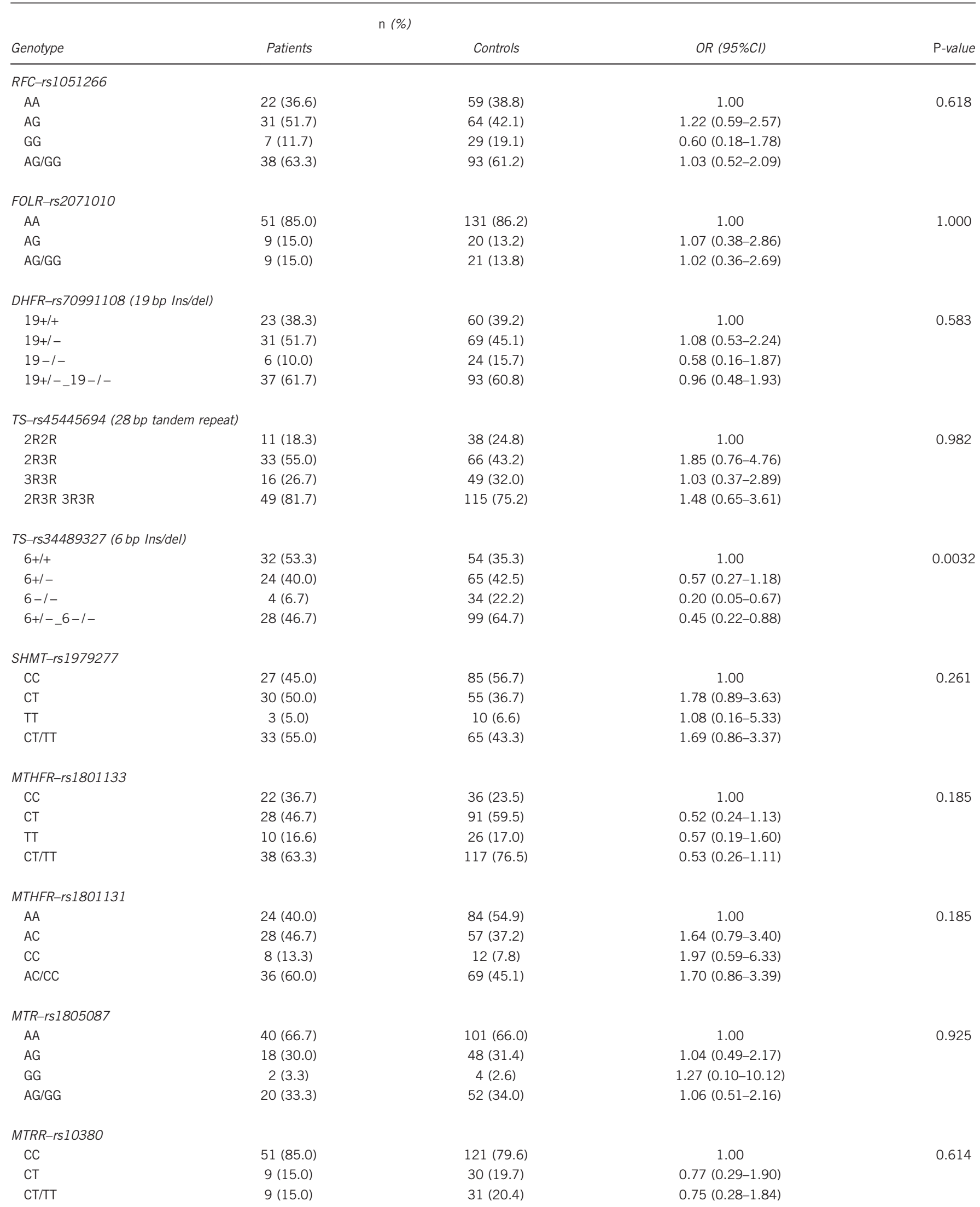


Table 1 (Continued)

n (\%)

\begin{tabular}{lccc} 
Genotype & Patients & Controls & OR (95\%Cl) \\
\hline MTRR-rs162036 & & & 1.00 \\
AA & $48(80.0)$ & $112(73.7)$ & $0.71(0.29-1.63)$ \\
AG & $11(18.3)$ & $38(25.0)$ & $2.16(0.03-4.38)$ \\
GG & $1(1.7)$ & $2(1.3)$ & $0.76(0.32-1.70)$ \\
AG/GG & $12(20.0)$ & $40(26.3)$ & 1.00 \\
MTRR-rs1801394 & & $56(36.6)$ & $1.06(0.50-2.27)$ \\
AA & $21(35.0)$ & $64(41.8)$ & $0.69(0.24-1.87)$ \\
AG & $30(50.0)$ & $33(21.6)$ & $0.93(0.46-1.91)$ \\
GG & $9(15.0)$ & $97(63.4)$ & 0.58 \\
AG/GG & $39(65.0)$ & & 1.00 \\
MTRR-rs1532268 & & $66(43.1)$ & $1.03(0.49-2.15)$ \\
AA & $26(43.3)$ & $66(43.1)$ & $1.07(0.36-2.97)$ \\
AG & $25(41.7)$ & $21(13.8)$ & $1.04(0.53-2.06)$ \\
GG & $9(15.0)$ & $87(56.9)$ & 0.968 \\
AG/GG & $34(56.7)$ & & \\
\hline
\end{tabular}

Exact logistic regression, age and gender adjusted.

Table 2 Most relevant results correlating mutational status and candidate genotypes

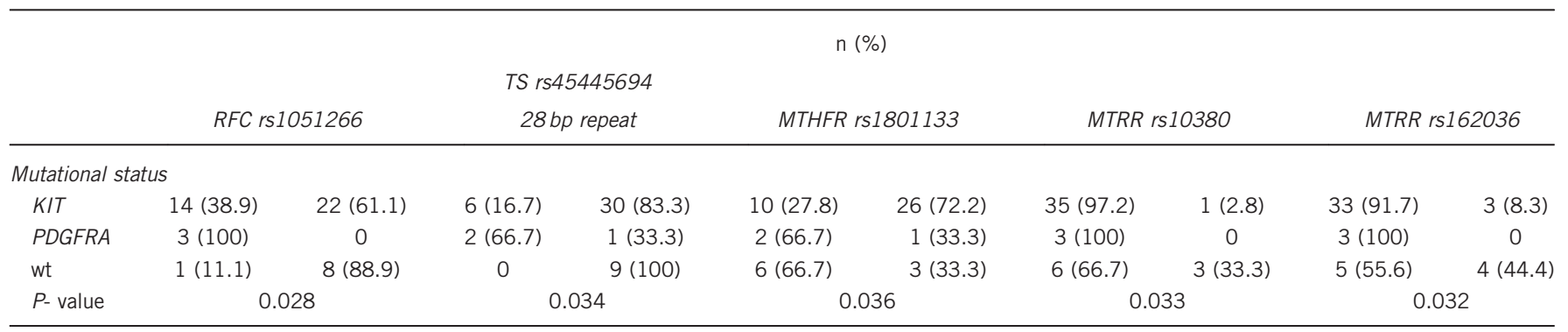

RFC allele was $0.24(95 \% \mathrm{CI}, 0.06-0.92 ; P=0.036$, age and gender adjusted). A similar influence on tumour size is exerted by MTHFR (rs1801133). Ten GIST patients (33.3\%) with at least one MTHFR variant allele had a tumour size larger than $10 \mathrm{~cm}$; in contrast, $70.6 \%$ patients with a wt MTHFR, $(68.4 \%)$ had a tumour size larger than $10 \mathrm{~cm}(\mathrm{OR}=0.21,95 \% \mathrm{CI}, 0.04-0.87 ; P=0.029$, age and gender adjusted). None of the other investigated polymorphisms showed any correlation with clinical features at diagnosis (data not shown).

\section{Association between TTP and genetic polymorphisms}

Fifty-four patients received standard first-line imatinib $400 \mathrm{mg}$ daily, with a median duration of imatinib administration of 36.9 months. Demographic and disease characteristics of this subgroup of GISTs have been extensively described elsewhere. ${ }^{16}$ Regarding imatinib response, the best results during therapy were partial response and stable disease in $90.7 \%$. With a median follow-up of 36.9 months, progression of disease was observed in 26 cases (48.1\%), with a median TTP of 21.8 (range 1.6-58.2). In the univariate analysis, we observed that the presence of the RFC variant allele (rs1051266) in homozygosis is associated, at the limit of statistical significance, with a reduced risk of disease progression $(\mathrm{HR}=0.144,95 \%$ CI $0.019-1.07$; $P=0.059$ ). The relationship resulted significant after correction for gender, age, and status at diagnosis $(\mathrm{HR}=0.107,95 \%$ CI $0.014-0.82$; $P=0.032$ ). Alleles were correlated with TTP based on the log-rank test. Presence of at least one wild-type allele in RFC (AA/AG) was associated with reduced TTP $(P=0.028)$. None of the other analysed polymorphisms correlated with the progression.

\section{Association between OS and genetic polymorphisms}

The genotype distribution of the analysed polymorphisms and the association with OS are summarised in Table 3. We observed a tendency towards an association between SHMT1 genotype and OS $(P=0.087)$. Compared with the homozygote SHMT1 CC genotype, the TT genotype was associated with a hazard of early death $(\mathrm{HR}=$ 6.53, 95\% CI 1.17-36.36). However, after a multivariate adjustment for gender, age, and status at diagnosis only a tendency for statistical significance of early death was observed ( $\mathrm{HR}=4.53$, 95\% CI $0.77-$ 26.58). None of the other analysed polymorphisms showed an association with the OS.

\section{DISCUSSION}

To the best of our knowledge this is the first study to investigate the association between tumours' characteristics and clinical outcomes and functional polymorphisms in genes of the folate pathway in GIST patients. Genetic approaches to define the mechanisms of GIST development, with the recognition of KIT/PDGFRA mutations as key players, have delivered meaningful insights into the development of treatment strategies. Nevertheless, imatinib remains the only first-line treatment approved, even in patients for whom we might anticipate a lack or a subsequent failure of efficacy. ${ }^{17}$ The mechanisms of 
Table 3 Univariate and multivariate analyses of the different genotypes and OS in GIST patients

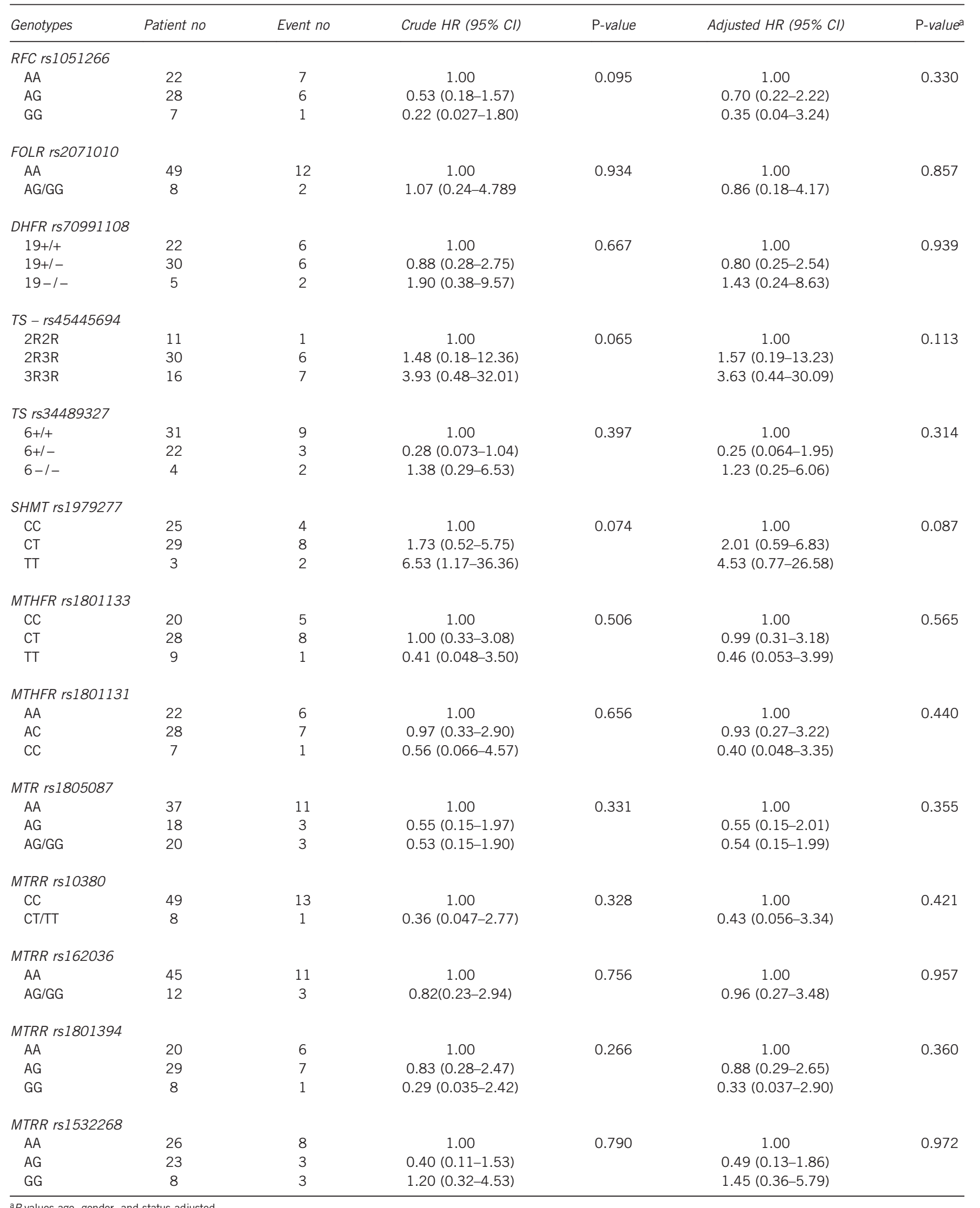

a $P$-values age, gender, and status adjusted. 
refractoriness or resistance, in addition to the acquisition of secondary mutations in KIT and PDGFRA, remain still unknown in most patients. ${ }^{18}$ We now recognise the enormous scope of genetic variation among humans that can be used to probe the genetics of treatment response and disease susceptibility. Basically all genes are subject to genetic variability, which can be associated with a person's risk for developing cancer, as a result of environmental exposures, as well as variability in drug response. Genetic polymorphisms in xenobiotic/drug metabolisers, transporters and targets loci were a natural starting point to study their relevance in susceptibility and treatment efficacy. Besides these, the biological components that may also influence disease susceptibility and therapies' outcome include enzymes that repair DNA damage, factors that regulate cell cycle control, cell division and cell death, and enzymes involved in the immune response. ${ }^{19}$ Many findings support the idea that different mechanisms may be involved in the GIST tumorigenesis processes, as emphasised by the widely different clinical behaviour of each GIST patient, regardless of the KIT/PDGFRA mutational status. In this regard we thought that polymorphisms in candidate genes related to the folate metabolism, involved in methylations, may be a good choice for investigating the association with clinicopathological features, response to treatment and survival. Our choice is supported by a recent finding of an aggressive clinical behaviour and unfavourable prognosis associated with DNA methylation profile in GIST, ${ }^{8}$ and is reinforced by the finding of a correlation between polymorphisms in folate genes and methylation status. ${ }^{6,7}$ In the present study the most remarkable result is the association with disease risk of the TS-rs34489327 polymorphism, a 6-bp ins/del in the 3' UTR region. The TS gene product, a folate-dependent enzyme, converting dUMP to dTMP, which is responsible for the incorporation of thymine during DNA synthesis. ${ }^{20}$ This variant, already associated with the occurrence of various tumours, such as colorectal cancer, lymphoma, and acute lymphocytic leukaemia, ${ }^{20-22}$ is believed to impair TS mRNA expression or stability, leading to reduced protein levels. ${ }^{23,24}$ The TS 6- bp del allele has been associated with a reduced enzymatic activity, resulting in depletion of deoxythymidine triphosphate (dTTP) pools followed by thymineless death or, in some instances, high levels of uracil misincorporation in DNA followed by extensive repair and subsequent double-strand breaks in DNA, that promote chromosomal instability, translocations, and aberrations. ${ }^{25}$ Interestingly, in our study the TS 6- bp deletion was found more frequently in the healthy controls, indicating a lower risk associated with the variant allele. Given the small population size, yet similar to other studies reported in the literature, owing to the rarity of the tumour, we cannot exclude that the association we found is by chance. Nevertheless, we should consider that the findings on cancer risk are controversial, with positive, negative or null results. ${ }^{25-32}$ Different hypotheses have been proposed to explain the observed discrepancies. It might reflect differences in the gene-disease association (that is, different types of cancer, different association) or may reflect different intakes of micronutrients, folate and $\mathrm{B}$ vitamins in particular, in the different populations. ${ }^{33-35}$ Alternatively, a further explanation is that TS requires 5,10-methylenetetrahydrofolate (5,10-MTHF) as a cofactor and competes with MTHFR for available 5,10-MTHF. The TS-mediated reaction is closely linked to the reaction catalysed by MTHFR, which implies a narrow interplay between these two enzymes. Therefore, addressing only polymorphisms in one gene may be an oversimplification of the reality and this prompted us to also include genetic polymorphisms in the key enzymes that take part in the folate metabolic pathways. However, none of the other investigated variants were associated with a higher risk of GIST. It should be noted that a better and meaningful approach would have been the gene-gene interactions analysis. However, this approach is not practical in our study, as it will necessarily suffer of insufficient statistical power owing to the small sample size.

Through our multiple-candidate gene approach, we found that polymorphism in RFC may be significantly associated with TTP. To us this finding is intriguing, as the gene product is involved in the folic acid cellular uptake and distribution. Previous studies have shown that individuals with the RFC-A allele, which is found significantly associated with reduced TTP in our study, had reduced plasma levels of folates and homocysteinaemia than individuals carrying the G allele. ${ }^{36,37}$ In turn, folate deficiency, may lead to inappropriate activation of proto-oncogenes and induction of malignant transformation through a mechanism of suboptimal DNA methylation. ${ }^{38}$ Therefore, it is plausible that the RFC genotype may be involved in GIST tumorigenesis, by affecting plasma folate levels.

Advances in molecular biology have highlighted that epigenetic modifications may have important roles in tumorigenesis and tumour progression. The different methylation status of several genes has been associated with different tumour phenotypes and clinical behaviours in quite a few cancers. ${ }^{39}$ Recently few studies have reported aberrant methylation status in GIST patients, particularly those with KIT/ PDGFRA wild type. ${ }^{40,41}$ As the methylation status of various genes greatly influences the diagnosis and prognosis of several tumours, it is reasonable to think that genetic polymorphisms in key enzymes of the folate metabolism may perturb this pathway and have the potential of becoming biomarkers of prognosis. To explore this hypothesis, we investigated the association of the selected genetic polymorphisms with OS. The SHMT variant resulted associated with OS. Because of the limitation of the present study due to the small sample size, results from survival analyses should be interpreted with care as model assumptions cannot be verified. SHMT is a vitamin B6-dependent SHMT1 enzyme that catalyses the reversible conversion of serine and tetrahydrofolate to glycine and 5,10-MTHF, needed for the synthesis of methionine, thymidylate, and purines. The $\mathrm{C}$ variant, associated with reduced $\mathrm{OS}$ in our population, has been associated with reduced folate levels; ${ }^{42,43}$ thus the polymorphism could mimic a situation of folate deficiency by limiting the availability of one-carbon units for both remethylation of homocysteine, important for DNA methylation, and DNA synthesis. However, it must be stressed that OS may be affected by multiple variables, and the potential prognostic role of the folate genotype should be verified in a larger sample size stratified according to the molecular and clinical features and the medical treatment received.

\section{CONCLUSION}

To conclude, we report significant association between genetic polymorphisms in key enzymes of the folate metabolic pathways and GIST tumorigenesis, clinical features and outcome. Our finding should be considered in the context of both the strengths - the investigation of a large number of polymorphisms across genes with well-defined roles in the folate pathway and the robust genotyping protocols - and limitations - the small sample size - and should be viewed as exploratory. On the other hand, the rarity of GIST requires that promising genetic polymorphisms, such as those reported in the present study, are subjected to further investigation. In particular, these results need to be further confirmed in larger independent studies, which will allow genome-wide association studies - only feasible when a large cohort of patients is available - opening up the opportunity to identify new loci associated with GIST susceptibility and/or clinical outcome, or to definitively confirm the role of candidate genetic variations. Furthermore, the enrichment of the study by having available serum folate levels or dietary intake, associated with the opportunity of gene-gene and gene-environment 
interaction analysis, may lead to a better understanding of GIST pathogenesis, clinical manifestation, and disease course.

\section{CONFLICT OF INTEREST}

The authors declare no conflict of interest.

\section{ACKNOWLEDGEMENTS}

This work was partially supported by the University of Bologna (Progetti strategici). Gloria Ravegnini is supported by a grant from Fondazione Umberto Veronesi - Post-Doctoral fellowship 2014. Muriel Musti has been supported by a grant from Fondazione del Monte di Bologna e Ravenna. The funders had no role in study design, data collection and analysis, or preparation of the manuscript.

1 Pogribny IP, Beland FA. DNA hypomethylation in the origin and pathogenesis of human diseases. Cell Mol Life Sci 2009; 66: 2249-2261.

2 Carr DF, Whiteley G, Alfirevic A, Pirmohamed M. FolATED study team: Investigation of inter-individual variability of the one-carbon folate pathway: a bioinformatic and genetic review. Pharmacogenomics J 2009; 9: 291-305.

3 Liu JJ, Ward RL. Folate and one-carbon metabolism and its impact on aberrant DNA methylation in cancer. Adv Genet 2010; 71: 79-121.

4 Fuso A. The "golden age" of DNA methylation in neurodegenerative disease. Clin Chem Lab Med 2013; 51: 523-534.

5 Nazki FH, Sameer AS, Ganaie BA. Folate: metabolism, genes, polymorphisms and the associated diseases. Gene 2014; 533: 11-20.

6 Coppedè F, Migheli F, Lopomo A et al: Gene promoter methylation in colorectal cancer and healthy adjacent mucosa specimens: correlation with physiological and pathological charcateristics, and with biomarkers of one-carbon metabolism. Epigenetics 2014; 9: 621-633.

7 Weiner AS, Boyarskikh UA, Voronina EN et al: Methylenetetrahydrofolate reductaseC677T and methionine synthase A2756G polymorphisms influence on leukocyte genomic DNA methylation level. Gene 2014; 533: 168-172.

8 Okamoto Y, Sawaki A, Ito $\mathrm{S}$ et al: Aberrant DNA methylation associated with aggressiveness of gastrointestinal stromal tumour. Gut 2012; 61: 392-401.

9 Hirota S, Isozaki K, Moriyama Y et al: Gain-of-function mutations of c-kit in human gastrointestinal stromal tumors. Science 1998; 279: 577-580.

10 Heinrich MC, Corless CL, Duensing $A$ et al: PDGFRA activating mutations in gastrointestinal stromal tumors. Science 2003; 299: 708-710.

11 Hayashi H, Fujimaki C, Daimon T, Tsuboi S, Matsuyama T, Itoh K. Genetic polymorphisms in folate pathway enzymes as a possible marker for predicting the outcome of methotrexate therapy in Japanese patients with rheumatoid arthritis. J Clin Pharm Ther 2009; 34: 355-361.

12 Johnson WG, Stenroos ES, Spychala JR, Chatkupt S, Ming SX, Buyske S. New 19 bp deletion polymorphism in intron-1 of dihydrofolate reductase (DHFR): a risk factor for spina bifida acting in mothers during pregnancy?. Am J Med Genet A 2004; 124A: 339-345.

13 Hishida $\mathrm{A}$, Matsuo $\mathrm{K}$, Hamajima $\mathrm{N}$ et al: Associations between polymorphisms in the thymidylate synthase and serine hydroxymethyltransferase genes and susceptibility to malignant lymphoma. Haematologica 2003; 88: 159-166.

14 Braun MS, Richman SD, Thompson L et al: Association of molecular markers with toxicity outcomes in a randomized trial of chemotherapy for advanced colorectal cancer: the FOCUS trial. J Clin Oncol 2009; 27: 5519-5528.

15 Stata Corporation. Stata Statistical software, release 11. College Station, TX: Stata Corporation 2011.

16 Angelini S, Pantaleo MA, Ravegnini G et al: Polymorphisms in OCTN1 and OCTN2 transporters genes are associated with prolonged time to progression in unresectable gastrointestinal stromal tumors treated with imatinib therapy. Pharmacol Res 2013; 68: 1-6.

17 Angelini S, Ravegnini G, Fletcher JA, Maffei F, Hrelia P. Clinical relevance of pharmacogenetics in GIST treatment in the era of personalized therapy". Pharmacogenomics 2013; 14: 941-956.

18 Maleddu A, Pantaleo MA, Nannini M et al: Mechanisms of secondary resistance to tyrosine kinase inhibitors in gastrointestinal stromal tumours. Oncol Rep 2009; 21: 1359-1366.

19 Upadhyay R, Jain M, Kumar S, Ghoshal UC, Mittal B. Association of interleukin-6 $(-174 G>C)$ promoter polymorphisms with risk of squamous cell esophageal cancer and tumor location: an exploratory study. Clin Immunol 2008; 128: 199-204.
20 Ulrich CM, Bigler J, Bostick R, Fosdick L, Potter JD. Thymidylate synthase promoter polymorphism, interaction with folate intake, and risk of colorectal adenomas. Cancer Res 2002; 62: 3361-3364.

21 Kawate H, Landis DM, Loeb LA. Distribution of mutations in human thymidylate synthase yielding resistance to 5-fluoro-deoxyuridine. J Biol Chem 2002; 277: 36304-36311.

22 Trinh BN, Ong CN, Coetzee GA, Yu MC, Laird PW. Thymidylate synthase: a novel genetic determinant of plasma homocysteine and folate levels. Hum Genet 2002; 111: 299-302.

23 Mandola MV, Stoehlmacher J, Zhang W et al: A 6bp polymorphism in the thymidylate synthase gene causes message instability and is associated with decreased intratumoral TS mRNA levels. Pharmacogenetics 2004; 14: 319-327.

24 Schwarzenbach H, Goekkurt E, Pantel K, Aust DE, Stoelmacher J. Molecular analysis of the polymorphisms of thymidylate synthase on cell-free circulating DNA in blood of patients with advanced colorectal carcinoma. Int J Cancer 2010; 127: 881-888.

25 Skibola CF, Forrest MS, Coppedé F et al: Polymorphisms and haplotypes in folatemetabolizing genes and risk of non-hodgkin lymphoma. Blood 2004; 104: 2155-2162

26 Graziano F, Kawakami K, Watanabe G et al: Association of thymidylate synthase polymorphisms with gastric cancer susceptibility. Int J Cancer 2004; 112: 1010-1014.

27 Lightfoot TJ, Skibola CF, Willett EV et al: Risk of non-Hodgkin lymphoma associated with polymorphisms in folate-metabolizing genes. Cancer Epidemiol Biomarkers Prev 2005; 14: 2999-3003.

28 Ulrich CM, Curtin K, Potter JD, Bigler J, Caan B, Slattery ML. Polymorphisms in the reduced folate carrier, thymidylate synthase, or methionine synthase and risk of colon cancer. Cancer Epidemiol Biomarkers Prev 2005; 14: 2509-2516.

29 Zhang Z, Xu Y, Zhou J et al: Polymorphisms of thymidylate synthase in the 5'- and 3'-untranslated regions associated with risk of gastric cancer in South China: a case-control analysis. Carcinogenesis 2005; 26: 1764-1769.

30 Zhai X, Gao J, Hu Z et al: Polymorphisms in thymidylate synthase gene and susceptibility to breast cancer in a Chinese population: a case-control analysis. BMC Cancer 2006; 6: 138.

$31 \mathrm{Kim}$ HN, Lee IK, Kim YK et al: Association between folate-metabolizing pathway polymorphism and non-hodgkin lymphoma. Brit J Haematol 2007; 140: 287-294.

32 Akisik E, Dalay N. Functional polymorphism of thymidylate synthase, but not of the COMT and IL-1B genes, is associated with breast cancer. J Clin Lab Analysis 2007; 21: 97-102.

33 Hubner RA, Liu JF, Sellick GS, Logan RF, Houlston RS, Muir KR. Thymidylate synthase polymorphisms, folate and B-vitamin intake, and risk of colorectal adenoma. Brit $J$ Cancer 2007; 97: 1449-1456.

34 Kawakita D, Matsuo K, Sato F et al: Association between dietary folate intake and clinical outcome in head and neck squamous cell carcinoma. Ann Oncol 2012; 23: 186-192.

35 Lucock M, Yates Z, Boyd L et al: Vitamin C-related nutrient-nutrient and nutrient-gene interactions that modify folate status. Eur J Nutr 2013; 52: 569-582.

36 Chango A, Emery-Fillon N, de Courcy GP et al: A polymorphism (80G->A) in the reduced folate carrier gene and its associations with folate status and homocysteinemia. Mol Genet Metab 2000; 70: 310-315.

37 Eklöf V, van Guelpen B, Hultdin J, Johansson I, Hallmans G, Palmqvist R. The reduced folate carrier (RFC1) 80G $>$ A and folate hydrolase 1 (FOLH1) 1561C $>$ T polymorphisms and the risk of colorectal cancer: a nested case-referent study. Scand J Clin Lab Invest 2007; 21: 1-9.

38 Wang L, Chen W, Wang J et al: Reduced folate carrier gene G80A polymorphisms is associatd with an increasd risk of gastroesophageal cancers in a Chinese population. Eur J Cancer 2006; 42: 3206-3211.

39 Esteller M, Corn PG, Baylin SB, Herman JG. Gene hypermethylation profile of human cancer. Cancer Res 2001; 61: 3225-3229.

40 Mason EF, Hornick JL. Succinate dehydrogenase deficiency is associated with decreased 5-hydroxymethylcytosine production in gastrointestinal stromal tumors: implications for mechanisms of tumorigenesis. Mod Pathol 2013; 26: 1492-1497.

41 Killian JK, Kim SY, Miettinen M et al: Succinate dehydrogenase mutation underlies global epigenomic divergence in gastrointestinal stromal tumor. Cancer Discov 2013; 3: 648-657.

42 Heil SG, Van der Put NM, Waas ET, den Heijer M, Trijbels FJ, Blom HJ. Is mutated serine hydroxymethyltransferase (SHMT) involved in the etiology of neural tube defects?. Mol Genet Metab 2001; 73: 164-172.

43 Relton CL, Wilding CS, Laffling AJ et al: Low erythrocyte folate status and polymorphic variation in folate-related genes are associated with risk of neural tube defect pregnancy. Mol Genet Metab 2004; 81: 273-281.

Supplementary Information accompanies this paper on European Journal of Human Genetics website (http://www.nature.com/ejhg) 